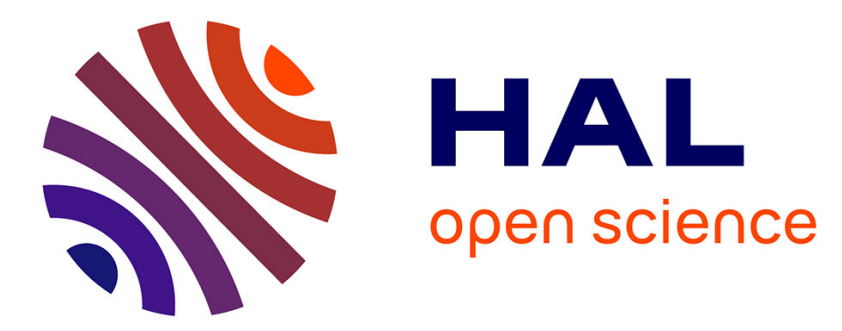

\title{
Influence of Kinetic Coupling in Rectilinear Coordinates on the Vibrational Spectrum of Fluoroform
}

Patrick Cassam-Chenaï, Yohann Scribano, Jacques Liévin

\section{To cite this version:}

Patrick Cassam-Chenaï, Yohann Scribano, Jacques Liévin. Influence of Kinetic Coupling in Rectilinear Coordinates on the Vibrational Spectrum of Fluoroform. Chemical Physics Letters, 2008, 466 (1-3), pp.16. 10.1016/j.cplett.2008.10.025 . hal-00315636

\section{HAL Id: hal-00315636 \\ https://hal.univ-cotedazur.fr/hal-00315636}

Submitted on 29 Aug 2008

HAL is a multi-disciplinary open access archive for the deposit and dissemination of scientific research documents, whether they are published or not. The documents may come from teaching and research institutions in France or abroad, or from public or private research centers.
L'archive ouverte pluridisciplinaire HAL, est destinée au dépôt et à la diffusion de documents scientifiques de niveau recherche, publiés ou non, émanant des établissements d'enseignement et de recherche français ou étrangers, des laboratoires publics ou privés. 


\title{
Influence of Kinetic Coupling in Rectilinear Coordinates on the Vibrational Spectrum of Fluoroform
}

\author{
Patrick Cassam-Chenaï, Yohann Scribano, \\ Laboratoire de Mathématiques J.A.Dieudonné, UMR CNRS 6621, Parc Valrose, \\ 06108 Nice Cedex 2, France \\ Jacques Liévin, \\ Université Libre de Bruxelles, Service de Chimie Quantique et Photophysique, CP \\ 160/09, 50 Av. F.D. Roosevelt, B-1050 Bruxelles, Belgium.
}

\begin{abstract}
The vibrational energy levels of Fluoroform have been computed with and without the kinetic coupling terms of the Eckart-Watson Hamiltonian by using the vibrational mean field configuration interaction method. The results are well-converged as demonstrated by a comparison with those obtained with other variational methods when kinetic coupling is omitted. It is found that kinetic coupling is not negligeable for this system. The wave number difference with and without kinetic coupling is $37 \mathrm{~cm}^{-1}$ for the $\nu_{1}$ (CH-streching) fundamental transition and can be larger than $60 \mathrm{~cm}^{-1}$ in the $\nu_{1}-\nu_{4}$ (HCF bending) overtones.
\end{abstract}

Key words: vibrational mean field configuration interaction, kinetic coupling operator, Fermi resonance.

PACS: 31.15.xr, 31.15.xt, 31.50.Bc, 33.20.Tp

Email addresses: cassam@unice.fr (Patrick Cassam-Chenaï), scribano@unice.fr (Yohann Scribano), jlievin@ulb.ac.be (Jacques Liévin).

Preprint $\quad$ for $\quad H A L$ 


\section{Introduction}

The quantum dynamics of the $\mathrm{CH}$ overtones of fluoroform $\mathrm{CF}_{3} \mathrm{H}$ has been studied experimentally [1] as well as theoretically [2-4] in order to characterize the intramolecular vibrational-energy redistribution (IVR) that follows excitation of the $\mathrm{CH}$ stretching mode. The vibrational degrees-of-freedom of the system can be partitioned into, on one hand, the $\mathrm{CH}$ stretching mode ( $\nu_{1}$-mode in spectroscopic notation) and the doubly degenerate $\mathrm{FCH}$ bending mode $\left(\nu_{4}\right.$-mode) which constitute the "chromophore" of the molecule, since the bright states correspond to excitation to these modes, and, on the other hand, the rest of the modes that constitutes the "bath" in which the chromophore is immersed. The theoretical description of the system is complicated because strong Fermi resonances couple states within the chromophore and because a network of anharmonic interactions connect the chromophore bright states with dark states of the bath. Moreover the presence of a light atom suggests that kinetic coupling is far from being negligible. So, describing the vibrational energy level pattern of such a system is quite challenging.

A correct understanding of the spectroscopy, dynamics and reactivity of such a molecule requires an accurate representation of the molecular quantum eigenstates. This in turn requires a reliable method to solve the molecular Schrödinger equation, and in particular, after decoupling of the internal degrees of freedom, an efficient method to solve the vibrational Schrödinger equation. Perturbative methods are not well suited for molecular systems with a high density of states or presenting resonances, and one has to turn oneself towards variational approaches.

One such approach, is the vibrational mean field configuration interaction (VMFCI) method [5-7] implemented in the CONVIV code that has been used in the present work. This flexible method has been shown to converge fundamental frequencies of $\mathrm{C}_{2} \mathrm{H}_{4} \mathrm{O}$, an hepta-atomic system [8] presenting strong resonances. Similar accuracy is out-of-reach of the traditional vibrational selfconsistent field (VSCF) $[9,10] /$ vibrational configuration interaction (VCI) (with virtuals from ground state VSCF calculations [11]) approach.

Another necessary condition for obtaining accurate vibrational quantum states, is to employ a good quality vibrational Hamiltonian, that is to say, within the Born-Oppenheimer approximation, a good quality potential energy function, and the relevant rotation-vibration kinetic couplings. We have used in this study on fluoroform the potential energy surface (PES) function of Maynard et al. [2], which has been used in many previous works, and can serve as a reference. The PES of Ramesh and Siebert [16] will be used in a forthcoming study. 
None of the previous studies on fluoroform using the Maynard et al. PES has taken into account the rotation-vibration kinetic couplings. The purpose of the present work is to show to which extend these kinetic couplings in rectilinear coordinates affect the vibrational energy levels.

The letter is organized as follows: First the VMFCI method is briefly outlined and the results obtained without kinetic couplings are compared with other methods for the same PES, in order to assess the convergence of the calculated wave numbers. Then, we show how the kinetic coupling terms of the EckartWatson Hamiltonian affect these wave numbers. Finally, we conclude on the importance of adding kinetic couplings terms in the fluoroform vibrational Hamiltonian.

\section{$2 \quad \mathrm{CF}_{3} \mathrm{H}$ vibrational states calculation}

\subsection{The VMFCI method for solving the vibrational Schrödinger equation}

Let us briefly sketch out the VMFCI method. The main idea behind it consists in calculating contracted basis sets for groups of degrees of freedom in order to obtain an all-degree basis set of managable size which captures the physics and can be used for performing a VCI calculation. However, in contrast with the usual contracted VCI approach [12-15] a mean field term is added to the group Hamiltonians and it has been demonstrated that it is the key of the success of the method of contraction [8]. In fact, the VMFCI approach contains the VSCF/VCI method as particular case, but it is much more general, as it allows one to perform successive contracted VCI for arbitrarily selected "active" degrees of freedom in the mean field of the other degrees called "spectator" degrees.

More precisely, at each VMFCI step, one starts with a partition, $P$, of the $n_{v i b}$ vibrational degrees of freedom of an oscillator system into $n_{P}$ subsets $I_{1}, I_{2}, \cdots, I_{n_{P}}$, of respectively $k_{1}, k_{2}, \cdots, k_{n_{P}}$ degrees:

$$
\begin{aligned}
P & =\left(I_{1}, I_{2}, \cdots, I_{n_{P}}\right) \\
& =\left(\left\{i_{1}^{1}, i_{2}^{1}, \cdots, i_{k_{1}}^{1}\right\},\left\{i_{1}^{2}, i_{2}^{2}, \cdots, i_{k_{2}}^{2}\right\}, \cdots,\left\{i_{1}^{n_{P}}, i_{2}^{n_{P}}, \cdots, i_{k_{n_{P}}}^{n_{P}}\right\}\right),
\end{aligned}
$$

and defines another partition, $S$, coarser than or equal to $P$, that is to say:

$$
S=\left(J_{1}, J_{2}, \cdots, J_{n_{S}}\right), \quad \text { with } \quad n_{S} \leq n_{P}
$$


and $\quad \forall j \in\left\{1, \cdots, n_{P}\right\}, \quad \exists k \in\left\{1, \cdots, n_{S}\right\} \quad$ such that, $\quad I_{j} \subseteq J_{k}$.

The subsets $J_{k}$ are referred to as "contractions" whose components are the $I_{j}$ 's such that $I_{j} \subseteq J_{k}$. The number of components in $J_{k}$ is denoted by $l_{k}$.

Assuming that:

(1) The vibrational Hamiltonian can be expanded in a factorized form:

$$
\begin{aligned}
H_{v i b}=h_{0} & +\sum_{j_{1}=1}^{n_{P}} h_{j_{1}}\left(I_{j_{1}}\right)+\sum_{1 \leq j_{1}<j_{2} \leq n_{P}} h_{j_{1}, j_{2}}\left(I_{j_{1}}\right) h_{j_{1}, j_{2}}\left(I_{j_{2}}\right) \\
& +\cdots+h_{1,2, \cdots, n_{P}}\left(I_{1}\right) h_{1,2, \cdots, n_{P}}\left(I_{2}\right) \cdots h_{1,2, \cdots, n_{P}}\left(I_{n_{P}}\right)
\end{aligned}
$$

where $h_{j_{1}, j_{2}, \cdots, j_{k}}\left(I_{j_{l}}\right)$ denotes a vibrational operator which only depends upon operators acting on the degrees in subset $I_{j_{l}}$,

(2) We have $n_{P}$ basis sets of vibrational wave functions, $\left\{\phi_{I_{j}}^{m}\left(q_{i_{1}^{j}}, \cdots, q_{i_{k_{j}}^{j}}\right)\right\}_{m \in\left\{1, d_{j}\right\}}$, with $1 \leq j \leq n_{P}$, spanning the Hilbert subspaces of dimension $d_{j}$, of physical interest for the groups of degrees of freedom in the $I_{j}$ 's,

the VMFCI calculation for that step consists in defining for each contraction, $J_{k}$, the mean field Hamiltonian obtained by averaging the vibrational Hamiltonian in the mean field of the other contractions $J_{l} \neq J_{k}$ and by diagonalizing it in a basis set of product functions,

$$
\Phi_{J_{k}}^{M}=\prod_{I_{j} \subseteq J_{k}} \phi_{I_{j}}^{m_{j}}\left(q_{i_{1}^{j}}, \cdots, q_{i_{k_{j}}^{j}}\right)
$$

where the superlabel $M$ stands for the sequence of indices $m_{j}$ of the component functions. That is to say, one first builds a partial Hamiltonian by gathering all the terms of $H_{v i b}$ that involve exclusively operators acting on the $I_{j} \subseteq J_{k}$,

$$
\begin{aligned}
H_{k}=h_{0} & +\sum_{j_{1} \text { such that }, I_{j_{1} \subseteq J_{k}}} h_{j_{1}}\left(I_{j_{1}}\right) \\
& +\sum_{j_{1}<j_{2} \text { such that }, I_{j_{1}}, I_{j_{2}} \subseteq J_{k}} h_{j_{1}, j_{2}}\left(I_{j_{1}}\right) h_{j_{1}, j_{2}}\left(I_{j_{2}}\right)+\cdots \\
& +\sum_{j_{1}<\cdots<j_{l_{k}}} \text { such that, } I_{j_{1}, \cdots, I_{j_{k}} \subseteq J_{k}} h_{j_{1}, \cdots, j_{l_{k}}}\left(I_{j_{1}}\right) \cdots h_{j_{1}, \cdots, j_{l_{k}}}\left(I_{j_{l_{k}}}\right),
\end{aligned}
$$

(where the last sum reduces in fact to only one term). Then, one averages $H_{v i b}-H_{k}$ over a given state, that we have chosen to be, in this work, the product of the ground state functions, $\prod_{I_{j} \nsubseteq J_{k}} \phi_{I_{j}}^{0}\left(q_{i_{1}^{j}}, \cdots, q_{i_{k_{j}}^{j}}\right)$, of all the com- 
ponents of the spectator contractions. We obtain the mean-field Hamiltonian :

$$
H_{k}+\left\langle\prod_{I_{j} \nsubseteq J_{k}} \phi_{I_{j}}^{0}\left(q_{i_{1}^{j}}, \cdots, q_{i_{k_{j}}^{j}}\right)\left|H_{v i b}-H_{k}\right| \prod_{I_{j} \nsubseteq J_{k}} \phi_{I_{j}}^{0}\left(q_{i_{1}^{j}}, \cdots, q_{i_{k_{j}}^{j}}\right)\right\rangle .
$$

This Hamiltonian is diagonalized in the basis set of all product functions of Eq.(4), or in a subset of the latter obtained by selecting the product functions such that the sum of the energies of their components is below a given threshold. Additionally, extra threshold can be applied to the energy of any component.

\section{2 $\mathrm{CF}_{3} \mathrm{H}$ rectilinear coordinate vibrational Hamiltonians}

Fluoroform belongs to the $\mathrm{C}_{3 v}$ symmetry point group. It has nine vibrational degrees of freedom and six normal modes. According to spectroscopic labelling, $\nu_{1}$ to $\nu_{3}$ ( $\mathrm{A}_{1}$ symmetry) correspond to $\mathrm{CH}$ stretching $\left(Q_{1}\right), \mathrm{CF}_{3}$ symmetric stretching $\left(Q_{2}\right)$ and $\mathrm{CF}_{3}$ symmetric deformation $\left(Q_{3}\right)$ respectively, and $\nu_{4}$ to $\nu_{6}$ (E symmetry) correspond to FCH bending $\left(Q_{4 a}, Q_{4 b}\right), \mathrm{CF}_{3}$ asymmetric stretching $\left(Q_{5 a}, Q_{5 b}\right)$ and $\mathrm{CF}_{3}$ asymmetric deformation $\left(Q_{6 a}, Q_{6 b}\right)$ respectively.

The Hamiltonians used in this study can be expressed as

$$
H_{v i b}=\sum_{k=1}^{6 b} P_{k}^{2}+P_{1} \cdot\left(\alpha P_{2}+\beta P_{3}\right)+V\left(Q_{1}, \ldots, Q_{6 b}\right)+W\left(Q_{1}, P_{1}, \ldots, Q_{6 b}, P_{6 b}\right) .
$$

In this expression, $V$ is the Born-Oppenheimer PES term. The PES used in this study has been calculated by Maynard et al. [2]. It is expressed in rectilinear coordinates, and uses a local coordinate description of the $\mathrm{CH}$ stretching and a normal coordinate description for the other modes. The 3D chromophore $\left(Q_{1}, Q_{4 a}, Q_{4 b}\right)$ is described by a $14^{\text {th }}$-order Taylor expansion while a fourthorder expansion is used for the other modes and intermode couplings. The resulting Born-Oppenheimer potential operator contains 467 terms, that have the form of a product of powers of the coordinates. In addition, the pure, local mode dimension is described by a Morse potential term. So, the potential is expanded as a sum of product terms as in equation (3), and is amenable to effective VMFCI calculations. This PES has been computed at the (6$\left.311 \mathrm{G}^{* *} / \mathrm{MP} 2\right)$ level of theory and the chromophore part was improved by using a more accurate method (cc-PVTZ/CEPA-1). The PES has been estimated [4] to be sufficiently accurate up to $9500 \mathrm{~cm}^{-1}$ excitation energy in the bath modes and up to $15000 \mathrm{~cm}^{-1}$ for the chromophore. 
In eq.(7), the $P_{k}$ are the conjugate momenta of the $Q_{k}$. Because, the local mode $Q_{1}$ is close to, but not exactly equal to the corresponding normal mode, a first order corrective term, coupling $P_{1}$ to the two other $A_{1}$-modes conjugate momenta appears in the Maynard et al. Hamiltonian [2].

The last term in eq. $(7)$ is the $(J=0)$-Watson-Eckart Hamiltonian rotationvibration kinetic coupling term,

$$
W\left(Q_{1}, P_{1}, \ldots, Q_{6 b}, P_{6 b}\right)=\frac{1}{2} \sum_{\alpha \beta} \mu_{\alpha \beta} \pi_{\alpha} \pi_{\beta}-\frac{1}{8} \sum_{\alpha} \mu_{\alpha \alpha},
$$

where $\pi$ is the so-called "Coriolis coupling operator" and $\mu$ is the $3 \times 3$ matrix derived from the inertia tensor. Rigorously speaking, the coordinates and conjugate momenta appearing in the latter two operators should be the rectilinear normal ones. However, since $W$ is only a corrective term and since the projection of the local mode coordinate on the normal $\mathrm{CH}$-stretch coordinate is close to one ( $\alpha$ and $\beta$ small in eq.(7)), the difference between the local and the normal $\mathrm{CH}$-mode has been neglected in $W$. This zeroth-order approximation is indeed consistent with the use of a first order approximation in the second term of eq. (7).

In practice, the $W$ term used in this study has been obtained by expanding the $\mu$-matrix in power of the $Q_{k}$,

$$
\mu=\sum_{n=0}^{+\infty}\left(\frac{1}{2}\right)^{n}(n+1) \times \sum_{k_{1}, \ldots, k_{n}} I_{e}^{-1} a_{k_{1}} I_{e}^{-1} \ldots a_{k_{n}} I_{e}^{-1} Q_{k_{1}} \ldots Q_{k_{n}}
$$

where $I_{e}^{-1}$ is the inverse of the inertia tensor $I\left(Q_{1}, \ldots, Q_{6 b}\right)$ at equilibrium and $\left(a_{k}\right)_{k}$ are the derivatives of the latter with respect to the normal coordinates at the equilibrium geometry (assuming that it is also the Eckart frame reference geometry, as is common practice),

$$
a_{k}=\left(\frac{\partial I}{\partial Q_{k}}\right)_{0}
$$

Previous studies using the Maynard et al. Hamiltonian have neglected $W$. This correspond to setting $\mu=0$. We call "order X" those calculations that use this $(\mu=0)$-Hamiltonian. Other calculations performed in this work have been obtained by truncating the expansion (9) at a given order, say $n$, and are called "order $n$ " calculations. 


\subsection{Selection of the VMFCI contraction scheme}

The VMFCI contraction scheme selected in this study starts as usual [7] with minimal symmetry preserving (MSP) steps which consist in contracting the degenerate modes together and only them, and which, as the name indicates, prevent artefactual symmetry breaking to occur. This is in contrast with the VSCF method which usually lift artefactually the degeneracy [17]. For fluoroform, MSP-VMFCI wavefunctions have the product form,

$$
\phi_{1}\left(Q_{1}\right) \phi_{2}\left(Q_{2}\right) \phi_{3}\left(Q_{3}\right) \phi_{4}\left(Q_{4 a}, Q_{4 b}\right) \phi_{5}\left(Q_{5 a}, Q_{5 b}\right) \phi_{6}\left(Q_{6 a}, Q_{6 b}\right) .
$$

The MSP partition has been iterated 6 times untill self-consistency was achieved to machine (double) precision, which means that a MSP vibrational selfconsistent field configuration interaction calculation (MSP-VSCFCI) has been performed.

Next, for selecting the contraction scheme of the MSP-VSCFCI basis set, we have calculated the ZPE value for all different two-mode contractions. They are reported in Table 1, the contractions being ordered by increasing ZPE values. Clearly, the most efficient contraction in terms of ZPE lowering is the $\left\{\nu_{1}-\nu_{2}\right\}$ contraction, whose ZPE lowering $\left(19 \mathrm{~cm}^{-1}\right)$ is an order of magnitude larger than the next one in the list $\left(2 \mathrm{~cm}^{-1}\right)$, in spite of the fact that only two degrees of freedom are involved, whereas other contractions can involve up to three or four degrees. So, on this ground, we have decided to contract modes $\left\{\nu_{1}-\nu_{2}\right\}$ straight after the MSP-VSCFCI.

Since in this study, we focus on the $\left\{\nu_{1}-\nu_{4}\right\}$ chromophore and since resonances between modes $\nu_{1}$ and $\nu_{4}$ excited states do not necessarily show up with the ZPE lowering criterium, we have actually opted for the ternary contraction $\left\{\nu_{1}-\nu_{2}-\nu_{4}\right\}$ which lowers the ZPE by $22 \mathrm{~cm}^{-1}$. We have not considered larger contraction than this 4-degrees contraction, because it would have been less computer effective given the limited RAM space of our computer: The integral file loaded in RAM at the next VMFCI step would require too drastic an energy truncation threshold to fit in.

Iterating the $\operatorname{VMFCI}\left(\nu_{1}-\nu_{2}-\nu_{4}\right)$ step once has been found to be sufficient to achieve self-consistency. Finally, the $\operatorname{VSCFCI}\left(\nu_{1}-\nu_{2}-\nu_{4}\right)$ has been followed by several all-degree VCIs differing from their truncation thresholds in the last step. 


\subsection{Convergence of the VMFCI calculations}

Table 2 reports the results obtained with the VMFCI contraction scheme described above for different truncation schemes at the last step. These calculations have been perfomed with the Hamiltonian, Eq. (7), and no rotationvibration kinetic coupling $(W=0)$. We have neglected the Eckart-Watson term, Eq. (8), to be able to compare our results with those obtained in other studies [2-4] where the same Hamiltonian was used.

The first column of Tab. 2 provides the assignement of the vibrational states in terms of harmonic functions or Morse potential eigenstates of highest weight as reported in previous studies [3,4]. However, these basis functions are highly mixed due to the presence of resonances as seen from the expansion of excited states such as $4 \nu_{4}, \nu_{1}+2 \nu_{4}$ and $2 \nu_{2}$ (see Tab.V of [3] and Tab. 3). In our $\operatorname{VSCFCI}\left(\nu_{1}-\nu_{2}-\nu_{4}\right)$ and VCI calculations, it is more natural to assign states in terms of the MSP-VSCFCI basis functions. Using the MSP-VSCFCI functions of highest weight, we find the same labelling for the vibrational states than that of Tab. 2 except for the excited states $4 \nu_{4}$ and $\nu_{1}+2 \nu_{4}$ which are inverted, moreover the $\nu_{1}+2 \nu_{4} \operatorname{VSCFCI}\left(\nu_{1}-\nu_{2}-\nu_{4}\right)$-eigenfunction has in fact its highest weight on the $2 \nu_{1}$ MSP-VSCFCI basis function (see Tab. 3).

The second column of Table 2 reports the harmonic approximation frequencies (modes $\nu_{2}$ to $\nu_{6}$ ) and for mode $\nu_{1}$ the Morse potential approximation. All VMFCI calculations start with a MSP-VSCFCI calculation using a basis set of harmonic oscillator (HO) functions with quantum numbers less than $60,40,40,40,40,60$, respectively, for the vibrational modes $\nu_{1}, \nu_{2}, \nu_{3}, \nu_{4}, \nu_{5}, \nu_{6}$, respectively. (Integrals of the Morse potential function between $\mathrm{HO}$ wave functions are calculated analytically in our code CONVIV by using the formulas of Ref. [18].) The results are reported in the third column and show large deviations with respect to the Harmonic/Morse approximation due to the anharmonicity of the system.

The next column correspond to the subsequent $\operatorname{VSCFCI}\left(\nu_{1}-\nu_{2}-\nu_{4}\right)$ with truncation of the product basis set on the sum of the energies at $24000 \mathrm{~cm}^{-1}$. In this calculation, the basis sets of the other modes have also been truncated at $24000 \mathrm{~cm}^{-1}$ for practical purposes, but this has no influence on the end results. We observe again large deviations for the contracted modes with respect to the MSP-VSCFCI results. In particular, the chromophore overtones change by more than $100 \mathrm{~cm}^{-1}$ for the $2 \nu_{1}$ and by as much as $300 \mathrm{~cm}^{-1}$ for the $4 \nu_{4}$. This fully justifies a posteriori the inclusion of mode $\nu_{4}$ in the contraction.

The VCI results (fifth column) have been obtained with a truncation on the sum of the energies at $10200 \mathrm{~cm}^{-1}$ and a truncation on the energy of mode $\nu_{6}$ at $6000 \mathrm{~cm}^{-1}$. The total number of basis functions is 67092 and the VMFCI 
scheme reads in rather self-explanatory notation (defined explicitly in ref. [7]), MSP-VSCFCI $(60,40,40,40,40,60) / \operatorname{VSCFCI}\left(\nu_{1}-\nu_{2}-\nu_{4} ; 24000\right) / \operatorname{VCI}(*, *, *, 6000 \mid 10200)$ (No truncation on a given contraction component is indicated by a "**" symbol.)

These results are to be compared with those reported in the last column obtained with the multi-configuration time dependent Hartree-Fock (MCTDH) approach ([4] and[19] for wave numbers below $3049 \mathrm{~cm}^{-1}$ ), which is also a variational method. These MCTDH wave numbers are very close to the wave operator sorting algorithm (WASO) wave numbers [3] not reported here. We see that the VCI and MCTDH ZPE are the same to the hundredth of $\mathrm{cm}^{-1}$ and that all tabulated VCI wave numbers are within $1 \mathrm{~cm}^{-1}$ of the MCTDH ones. In the bottom part of the spectrum, they are within the tenth of $\mathrm{cm}^{-1}$ of the MCTDH ones. This good agreement allows us to be confident in the reliability of our VMFCI scheme for the assessment of the rectilinear coordinates, rotation-vibration kinetic couplings which have not been considered in previous variational studies so far. The evaluation of these couplings will be the topics of the next section.

The effect of the VCI truncation thresholds on the tabulated wave numbers are investigated in Table 4. The two middle columns correspond to the same threshold of $10000 \mathrm{~cm}^{-1}$ on the sum of the components function energies in product basis functions. In addition, a threshold of $6000 \mathrm{~cm}^{-1}$ is applied on the $\nu_{6}$-component function energy in the calculation presented in the second column. This reduces the basis set size by 2312 basis function. However, the tabulated wave numbers are identical in the two columns except for $4 \nu_{4}$ where they differ by $0.1 \mathrm{~cm}^{-1}$. So, including excited $\nu_{6}$ MSP-VSCFCI basis function is not crucial for the eigenstates of interest and the use of a threshold on the $\nu_{6}$-component function energy is justified in Tab.2.

Columns 1, 2 and 4 of Tab. 4 shows the convergence pattern with respect to the threshold on the sum of the component energies. The bottom of the spectrum is already well converged with a threshlod of $9800 \mathrm{~cm}^{-1}$ and less than 53000 basis functions. Only the three levels of the chromophore $2 \nu_{1}$-polyad are not yet converged. The threshold at $10000 \mathrm{~cm}^{-1}$ adds 6722 basis functions and improves the polyad wave numbers significantly if the calculation with the $10200 \mathrm{~cm}^{-1}$ threshold serves as a reference. On the basis of the wave number convergence pattern, one can reasonably expect to have reached the $\mathrm{cm}^{-1}$ accuracy with the latter calculation. 


\section{Effect of the rectilinear coordinate rotation-vibration kinetic cou- pling}

Vibrational levels computed at different orders of $\mu$-matrix expansion are reported in Table 5. Order X corresponds to column 3 of Tab. 4. The results for the other orders of $\mu$-matrix expansion have been obtained with the same contraction-truncation scheme. It is clear that the $(W=0)$-Hamiltonian is not satisfactory because some fundamental frequencies are away from those of order 1 by more than the $1 \mathrm{~cm}^{-1}$. The largest errors are observed for the $\nu_{2}$ fundamental wave number $\left(\approx 16 \mathrm{~cm}^{-1}\right)$ and for $\nu_{1}\left(\approx 37 \mathrm{~cm}^{-1}\right)$. For higher excited states, the discrepancies grow even larger: $\approx 56 \mathrm{~cm}^{-1}$ for $4 \nu_{4}, \approx 62$ $\mathrm{cm}^{-1}$ for $\nu_{1}+2 \nu_{4}$, and $\approx 58 \mathrm{~cm}^{-1}$ for $2 \nu_{1}$.

In contrast, the results show that Order 0 is sufficient to take into account the kinetic coupling effect. The difference between Order 0 and Order 1 is equal to less than the tenth of $\mathrm{cm}^{-1}$ for all vibrational levels, (the errors of $0.1 \mathrm{~cm}^{-1}$ appearing in the table are due to round off of the last digits).

The inclusion of the kinetic coupling can bring some vibrational frequencies closer to the experimental ones [20] like for mode $\nu_{2}$ but this is not general. This hints to the fact that a better description of the PES is required to improve the agreement with the experimental data.

\section{Conclusion}

In this study, we have performed large variational calculations of the vibrational eigenstates of fluoroform by using an efficient vibrational Schrödinger equation solver, the VMFCI method. The accuracy of the eigenstates obtained have been checked against MCTDH results. Then, we have investigated the importance of the rotation-vibration kinetic coupling in rectilinear coordinates on the spectrum calculation. We have shown that it is not negligeable, however a zero order expansion of the $\mu$-matrix is sufficient to account for the coupling.

A natural follow-up to the present work will be the evaluation of the influence of the rotation-vibration kinetic coupling terms on intramolecular vibrational energy redistribution in highly excited fluoroform. 


\section{Acknowledgments}

We are grateful to Prof. C. Iung for providing us with a file containing the data corresponding to the PES of Maynard et al., and to Dr. J.-M. Chiaramello for his help in transforming the format of these data into a form readable by the code CONVIV. We thank Dr. F. Gatti for communicating his MCTDH results and allowing us to make use of them in this paper. This work was supported by the ANR "chaire d'excellence Persi Diaconis" who provided a postdoc position to one of us (YS) and by the ANR "projet AHBE". J.L. acknowledges the "Fonds National de la Recherche Scientifique de Belgique (contracts FRFC and IISN) and the "action de recherche concerté de la Communauté française de Belgique" for financial support. 


\section{References}

[1] O. Boyarkin, M. Kowalszyk and T. Rizzo, J. Chem. Phys 118 (2003) 93.

[2] A. T. Maynard, R. E. Wyatt and C. Iung, J. Chem. Phys. 103 (1995) 8372

[3] A. T. Maynard, R. E. Wyatt and C. Iung, J. Chem. Phys. 106 (1997) 9483

[4] C. Iung and F. Gatti, H. D. Meyer, J. Chem. Phys. 120 (2004) 6992

[5] P. Cassam-Chenaï and J. Liévin, Int. J. Quantum Chem. 93 (2003) 245-264.

[6] P. Cassam-Chenaï, J. Quant. Spectrosc. Radiat. Transfer 82 (2003) 251-277.

[7] P. Cassam-Chenaï, J. Liévin, Journal of Comp. Chem. 27 (2006) 627-640.

[8] D. Bégué, C. Pouchan, N. Gohaud, P. Cassam-Chenaï, J. Liévin, J. Chem. Phys 127 (2007) 164115-164124.

[9] J. M. Bowman, J. Chem. Phys 68 (1978) 608.

[10] G. D. Carney, L. L. Sprandel and C. W. Kern in "Advances in Chemical Physics", Vol. 37, I. Prigogine and S.A. Rice, Eds., (Wiley, New York, 1978), pp. 305-379.

[11] K. M. Christoffel, J. M. Bowman, Chem. Phys. Lett. 85 (1982) 220.

[12] C. L. Chen, B. Maessen, and M. Wolfsberg, J. Chem. Phys 83 (1985) 1795.

[13] J. Tennyson, Comp. Phys. Rep. 4 (1986) 1.

[14] S. Carter and N. C. Handy, Comp. Phys. Rep. 5 (1986) 15.

[15] Z. Bačić and J. C. Light, Annu. Rev. Phys. Chem. 40 (1989) 469.

[16] S. G. Ramesh and E. L. Sibert, Mol. Phys. 103 (2005) 149.

[17] R. Burcl, S. Carter, N. C. Handy, Chem. Phys. Lett. 373 (2003) 357-365.

[18] P. Palting, S. T. Lai, Y. N. Chiu, J. R. Letelier, J. Math. Chem. 21 (1997) 31.

[19] F. Gatti, private communication

[20] H. R. Dübal and M. Quack, J. Chem. Phys. 81 (1984) 3779 


\begin{tabular}{cccccccc}
\hline \hline$\nu_{1}-\nu_{2}$ & $\nu_{1}-\nu_{4}$ & $\nu_{1}-\nu_{3}$ & $\nu_{1}-\nu_{6}$ & $\nu_{4}-\nu_{5}$ & $\nu_{4}-\nu_{6}$ & $\nu_{2}-\nu_{4}$ & $\nu_{2}-\nu_{3}$ \\
\hline 5648.68 & 5665.31 & 5665.70 & 5665.92 & 5666.77 & 5667.13 & 5667.23 & 5667.38 \\
$\nu_{3}-\nu_{5}$ & $\nu_{3}-\nu_{6}$ & $\nu_{5}-\nu_{6}$ & $\nu_{3}-\nu_{4}$ & $\nu_{1}-\nu_{5}$ & $\nu_{2}-\nu_{6}$ & $\nu_{2}-\nu_{5}$ & \\
\hline 5667.44 & 5667.48 & 5667.57 & 5667.59 & 5667.60 & 5667.60 & 5667.60 & \\
\hline \hline
\end{tabular}

Table 1

Zero point energy in $\mathrm{cm}^{-1}$ after a MSP-VSCFCI $(60,40,40,40,40,60)$ calculation followed by two-mode contractions. The ZPE are arranged in increasing order. For comparison, the MSP-VSCFCI ZPE is $5667.61 \mathrm{~cm}^{-1}$. The product basis functions for the two-mode contractions have been truncated at $11400 \mathrm{~cm}^{-1}$ on the sum of the component energies. 


\begin{tabular}{cccccc}
\hline \hline assign. & Morse/HO & MSP-VSCFCI & VSCFCI $\left(\nu_{1}-\nu_{2}-\nu_{4}\right)$ & VCI & MCTDH $^{\mathrm{a}}$ \\
\hline $\mathrm{ZPE}$ & 5701.83 & 5667.61 & 5645.61 & 5638.54 & 5638.54 \\
$\nu_{6}$ & 518.2 & 509.4 & 509.4 & 507.0 & 507.0 \\
$\nu_{3}$ & 724.3 & 712.9 & 712.9 & 703.7 & 703.7 \\
$\nu_{2}$ & 1203.6 & 1200.9 & 1167.9 & 1157.8 & 1157.8 \\
$\nu_{5}$ & 1206.0 & 1190.3 & 1190.0 & 1175.8 & 1175.8 \\
$\nu_{4}$ & 1431.0 & 1416.6 & 1407.4 & 1396.2 & 1396.2 \\
$2 \nu_{4}$ & 2861.9 & 2862.6 & 2777.2 & 2750.0 & 2749.7 \\
$\nu_{1}$ & 3069.6 & 3014.0 & 3057.4 & 3048.9 & 3048.7 \\
$4 \nu_{4}$ & 5723.9 & 5789.3 & 5489.9 & 5435.1 & 5436 \\
$\nu_{1}+2 \nu_{4}$ & 5931.5 & 5876.6 & 5804.6 & 5758.0 & 5759 \\
$2 \nu_{1}$ & 6013.8 & 5899.3 & 6009.3 & 5988.2 & 5989 \\
\hline \hline
\end{tabular}

Table 2

Energies in $\mathrm{cm}^{-1}$ of the vibrational fundamental transitions and of the overtones of the $\left(\nu_{1}-\nu_{4}\right)$-chromophore calculated at different VMFCI levels (see text for details) and with the MCTDH algorithm. The tabulated energies of the $2 \nu_{4}, \nu_{1}+2 \nu_{4}, 4 \nu_{4}$ bands are those of their non degenerate levels.

$\overline{\text { a Ref. [4] }}$, and [19] for wave numbers below $3049 \mathrm{~cm}^{-1}$ 


\begin{tabular}{ccccc}
\hline \hline & $\operatorname{VSCFCI}\left(\nu_{1}-\nu_{2}-\nu_{4}\right)$ & $\left|\Phi_{4 \nu_{4}}^{\mathrm{MSP}}\right\rangle$ & $\left|\Phi_{\nu_{1}+2 \nu_{4}}^{\mathrm{MSP}}\right\rangle$ & $\mid \Phi_{2 \nu_{1}}^{\mathrm{MSP}}>$ \\
\hline $4 \nu_{4}$ & 5489.9 & $\mathbf{0 . 3 9}$ & $\mathbf{0 . 4 6}$ & 0.06 \\
$\nu_{1}+2 \nu_{4}$ & 5804.6 & 0.38 & 0.10 & $\mathbf{0 . 4 6}$ \\
$2 \nu_{1}$ & 6009.3 & 0.15 & 0.37 & $\mathbf{0 . 4 2}$ \\
\hline \hline
\end{tabular}

Table 3

Projection weights of contracted $\operatorname{VSCFCI}\left(\nu_{1}-\nu_{2}-\nu_{4}\right)$ eigenfunctions of Tab. 2 on relevant MSP basis functions. 


\begin{tabular}{|c|c|c|c|c|}
\hline assign. & $\begin{array}{c}\text { VMFCI }(9800) \\
52964 \text { bf. }\end{array}$ & $\begin{array}{l}\text { VMFCI(10000) } \\
59686 \text { bf. }\end{array}$ & $\begin{array}{c}\operatorname{VMFCI}^{(\star)}(10000) \\
61998 \text { bf. }\end{array}$ & $\begin{array}{c}\text { VMFCI(10200) } \\
67092 \text { bf. }\end{array}$ \\
\hline $\mathrm{ZPE}$ & 5638.54 & 5638.54 & 5638.54 & 5638.54 \\
\hline$\nu_{6}$ & 507.0 & 507.0 & 507.0 & 507.0 \\
\hline$\nu_{3}$ & 703.7 & 703.7 & 703.7 & 703.7 \\
\hline$\nu_{2}$ & 1157.8 & 1157.8 & 1157.8 & 1157.8 \\
\hline$\nu_{5}$ & 1175.8 & 1175.8 & 1175.8 & 1175.8 \\
\hline$\nu_{4}$ & 1396.3 & 1396.3 & 1396.3 & 1396.2 \\
\hline $2 \nu_{4}$ & 2750.1 & 2750.0 & 2750.0 & 2750.0 \\
\hline$\nu_{1}$ & 3049.0 & 3048.9 & 3048.9 & 3048.9 \\
\hline $4 \nu_{4}$ & 5439.8 & 5436.4 & 5436.3 & 5435.1 \\
\hline$\nu_{1}+2 \nu_{4}$ & 5761.5 & 5759.7 & 5759.7 & 5758.0 \\
\hline $2 \nu_{1}$ & 5989.5 & 5990.2 & 5990.2 & 5988.2 \\
\hline
\end{tabular}

Table 4

Convergence of vibrational levels with different VCI basis set truncation scheme. VMFCI actually stands for the contraction scheme: MSP$\operatorname{VSCFCI}(60,40,40,40,40,60) / \operatorname{VSCFCI}\left(\nu_{1}-\nu_{2}-\nu_{4} ; 24000\right) / \mathrm{VCI}$, and the value in parenthesis is the threshold in $\mathrm{cm}^{-1}$ applied to the sum of the energies of the product function components for the VCI. For all calculations, a truncation at $6000 \mathrm{~cm}^{-1}$ was applied to the energy of mode $\nu_{6}$ component except for $\mathrm{VMFCI}^{(\star)}(10000)$. "bf." stands for basis functions. 


\begin{tabular}{ccccc}
\hline \hline assign. & Ord X & Ord 0 & Ord 1 & Expt $^{\mathrm{b}}$ \\
\hline ZPE & 5638.54 & 5652.28 & 5652.28 & - \\
$\nu_{6}$ & 507.0 & 507.1 & 507.1 & 507.8 \\
$\nu_{3}$ & 703.7 & 703.6 & 703.6 & 700.1 \\
$\nu_{2}$ & 1157.8 & 1141.6 & 1141.7 & 1141.5 \\
$\nu_{5}$ & 1175.8 & 1176.5 & 1176.5 & 1158.3 \\
$\nu_{4}$ & 1396.3 & 1398.4 & 1398.5 & 1377.5 \\
$2 \nu_{4}$ & 2750.0 & 2768.2 & 2768.2 & 2710.2 \\
$\nu_{1}$ & 3048.9 & 3086.1 & 3086.1 & 3035.5 \\
$4 \nu_{4}$ & 5436.3 & 5492.0 & 5492.1 & 5337 \\
$\nu_{1}+2 \nu_{4}$ & 5759.7 & 5821.5 & 5821.6 & 5710.4 \\
$2 \nu_{1}$ & 5990.2 & 6048.3 & 6048.4 & 5959.4 \\
\hline \hline
\end{tabular}

Table 5

Energies in $\mathrm{cm}^{-1}$ of fluoroform vibrational states calculated at different order of $\mu$ matrix expansion (Order X corresponds to setting $\mu=0$ ). The tabulated values are obtained from a VCI at the last step with a truncation on the sum of the energies of product function components at $10000 \mathrm{~cm}^{-1}$ and are expected to be converged to the $\mathrm{cm}^{-1}$ precision.

b Ref. [20] 